EPJ Web of Conferences 71, 00079 (2014)

DOI: 10.1051/epjconf/20147100079

(C) Owned by the authors, published by EDP Sciences, 2014

\title{
Infinite symmetry in the quantum Hall effect
}

\author{
C.A. Lütken ${ }^{1,2, a}$ \\ ${ }^{1}$ Theory Group, Department of Physics, University of Oslo \\ ${ }^{2}$ Theory Group, CERN
}

\begin{abstract}
The new states of matter and concomitant quantum critical phenomena revealed by the quantum Hall effect appear to be accompanied by an emergent modular symmetry. The extreme rigidity of this infinite symmetry makes it easy to falsify, but two decades of experiments have failed to do so, and the location of quantum critical points predicted by the symmetry is in increasingly accurate agreement with scaling experiments. The symmetry severely constrains the structure of the effective quantum field theory that encodes the low energy limit of quantum electrodynamics of $10^{10}$ charges in two dirty dimensions. If this is a non-linear $\sigma$-model the target space is a torus, rather than the more familiar sphere. One of the simplest toroidal models gives a critical (correlation length) exponent that agrees with the value obtained from numerical simulations of the quantum Hall effect.
\end{abstract}

\section{Hall's effect}

A fact that never fails to baffle our students is that a charge $(Q)$ moving in a magnetic field $(B)$ feels a force that is orthogonal to both the field and the velocity $(v)$ of the charge. Therefore, when an electric current $I$ flows through a piece of conducting matter, usually a rectangular slab that is placed between the poles of a strong magnet (compare Fig. 1(a)), an electric potential develops transverse to the current. This is the classical Hall effect, named after the diligent graduate student who first succeeded in measuring this potential in 1879 [1].

The size of the transverse potential is inversely proportional to the thickness of the slab. Edwin Hall succeeded because he used gold leaf, which is two orders of magnitude thinner than the ordinary metal sheets used by his predecessors. However, hammered gold leaf is still thousands of atoms thick, so he could not observe the stunning quantum Hall effect, since this only appears in two dimensions.

A century later (1980) Klaus von Klitzing repeated Hall's experiment on a sandwich of semiconductors that forces the charges to move along the interface of two layers [2]. In essence his team had manufactured Edwin Abbott Abbott's famous mathematical Flatland - originally conceived in 1884 as a political satire on Victorian England. It soon became clear that the laws of nature in this physical 2-dimensional "designer universe" are very different from our 3-dimensional world.

As expected, at room temperature the classical Hall effect appeared: the ordinary direct (longitudinal) resistance $R_{D}=V_{D} / I$ is a finite material constant independent of $B$ (as expected from Ohm's law), and the transverse Hall resistance $R_{H}=V_{H} / I$ is proportional to $B$ (as expected from the magnetic force law $Q v \times B$ ). But when the chip was cooled to around 1 Kelvin von Klitzing unwittingly

ae-mail: lutken@fys.uio.no 

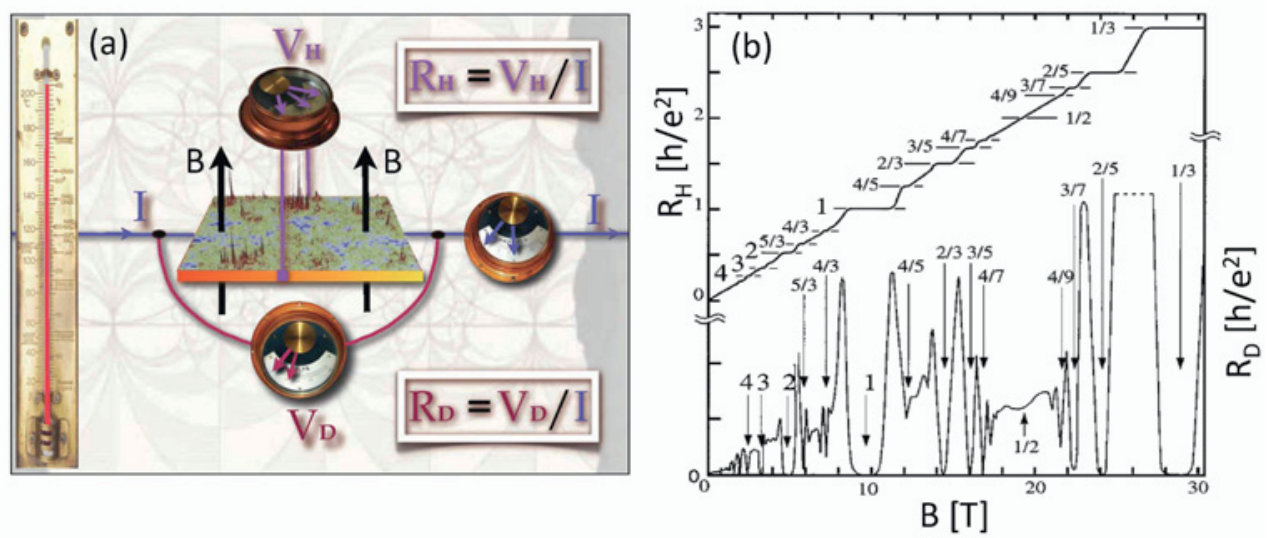

Figure 1. (Color online) (a) Hall's device (1879). On a surface at sufficiently low temperature, in a strong magnetic field at a critical value, the wave-function has support everywhere, and it may be multi-fractal as shown here. The backdrop is a modular diagram constructed by Fricke and Klein in 1886, similar to the phase- and flow-diagrams for the QHE to be discussed here. (b) Fixed temperature quantum Hall data from around year $2000[4]$.

stepped through a quantum looking glass, and what he found there was baffling new physics that continues to strain our understanding of quantum mechanics. Both resistances are "flattened" (compare Fig. 1(b)): for most values of the magnetic field $R_{D}$ vanishes and $R_{H}$ is piece-wise constant. This is the famous quantum Hall effect (QHE).

The first surprise is not that $R_{D}$ vanishes, but rather that it does not always vanish. Because conductance is given by $G_{D}=R_{D} /\left(R_{D}^{2}+R_{H}^{2}\right.$ ), as long as $B \neq 0$ (so $R_{H} \neq 0$ ), there is no conduction (charge transport) in the direction of the current when $R_{D}=0$. This is as expected, because cold charges in two dimensions should be localised, i.e., pinned to the dirt that is always contaminating any real crystal. The spikes in $R_{D}$ at certain critical values of $B$ means that the wave-function is delocalised (so it can carry charge across the sample), and signals the onset of a quantum phase transition between two distinct types of incompressible quantum liquids.

At these critical field strengths the Hall resistance "jumps" from one constant value to the next, creating a "Devil's staircase". Measured in the fundamental unit of resistance $R_{\mathrm{K}}=h / e^{2}=$ $25.812807557(18) k \Omega \approx 26 k \Omega$ ( $h$ is Planck's constant, and $e$ is the electron charge), the steps observed by von Klitzing are quantized at inverse integer values (IQHE): $R_{\mathrm{H}}=1 / p\left[R_{\mathrm{K}}\right](p \in \mathbb{Z})$. These integers can be simply understood as "spectroscopy" of Landau levels, but the second big surprise is how accurate this quantisation is, essentially exact (parts per billion) with current technology. This is why the IQHE today is used to define the Ohm $(\Omega)$. It also provides one of the most accurate measurements of the fine structure constant $(\alpha)$.

Two years later we got a third huge surprise: Daniel C. Tsui and Horst L. Störmer discovered fractionally quantised Hall plateaux [3]: $R_{\mathrm{H}}=q / p\left[R_{\mathrm{K}}\right](p \in \mathbb{Z}, q \in 2 \mathbb{Z}+1)$. It is conventional to label plateaux by their conductivity $p / q\left[e^{2} / h\right]$, as shown in Fig. 1(b). The odd parity of the denominator derives from the Fermi statistics of electrons, as shown by Robert B. Laughlin, who also explained how the exact quantisation observed by von Klitzing is required by the exact $U(1)$ gauge symmetry of Maxwell's unified theory of electricity and magnetism. The fractional quantum Hall effect (FQHE) was totally unexpected and heralded a brave new world of quantum weirdness. 
The importance of these discoveries was immediately recognised by the community, who rewarded von Klitzing, Laughlin, Störmer and Tsui with Nobel Prizes in 1985 and 1998. Three decades after their discovery we are still struggling to understand these new phases of condensed matter, the quantum phase transitions separating them, and the effective quantum field theory needed to understand the scaling, universality and critical properties of these systems.

\section{The quantum Hall problem}

The Hall plateaux can to some extent be understood by constructing simplified wave-functions for the charge carriers participating in the collective mode whose transport properties are observed in the experiments. It is clear that these sanitized many-particle wave-functions have virtually no overlap with the ground state of the real, physical, microscopic Hilbert space of $10^{10}$ electrons in a dirty environment ravaged by dislocations and impurities. Nevertheless, this construction works very well for the plateaux because of universality, one of the deepest and most useful concepts in physics (and indeed in all of science, albeit under different names).

The crux of this idea is that "less is more": very often we are not interested in all the microscopic details of the system, but rather the collective behaviour that emerges at scales where the "building blocks" (particles, atoms, molecules, cells,...) are unobservable, and by peeling off the irrelevant stuff we get a much clearer picture of what is going on. In short, less detail gives more insight.

The infinitely many microscopic Hamiltonians that give rise to the same macroscopic collective behaviour is called a universality class, and it is an empirical fact that we do not need very many of these to give a coherent description of nature, at any given scale. There are various ways of studying these universality classes, but it is always the simplest member that is most useful.

The idealised Hall Hamiltonian, whose ground state is Laughlin's first quantised wave-function, is a good example, since it captures the essence of the incompressible quantum Hall fluid at a plateaux in the simplest possible way. However, near critical points controlling phase transitions between plateaux it is not useful, and a different effective description is needed.

The appropriate language for discussing universality, scaling, phase transitions and critical exponents in a systematic manner is field theory, and we define the quantum Hall problem to be the construction of this effective quantum field theory. It is a problem because we do not know how to derive the low energy limit of quantum electrodynamics (QED) in two dirty dimensions. Simplified model wave-functions may be useful at the plateaux, but the plateaux are a set of zero measure in the parameter space of the quantum field theory, so they are of no assistance in this quest. A new approach is needed.

Similar problems arise in high energy physics, where we do not know how to derive the low energy limit of quantum chromodynamics (QCD). Quarks and self-interacting gauge bosons give rise to an insoluble set of highly non-linear differential equations. One of the earliest and most successful methods developed to circumvent this problem used to be called phenomenology, a precursor of what today is called effective field theory (EFT) [5].

The essence of this idea is to use some of the global properties of the theory - in the case of low energy QCD the geometry of flavour symmetries that are observed in the hadronic spectrum. These are approximate and may be broken, giving rise to pseudo-Goldstone bosons (pions), but they do suffice to construct an effective non-linear $\sigma$-model that successfully describes the behaviour of the low energy "lumps" (pions and nucleons), without any reference to the sub-stratum of quarks and gluons. The phenomenological approach to the QHE [6] (described below) is similar in spirit, since it exploits emergent symmetries to harness the phenomenology of the system, but the symmetries are very different. 
Notice that although we understand the microscopic foundations (QCD and QED) of both of these phenomenological models, in neither case do we know how to rigorously derive the effective theory from the microscopic one, and clearly we do not have to. Fortunately the world is foliated into layers of distinctive structures, and this separation of scales allows us to peel the cosmic onion one layer at a time. If we demanded that every layer be derived from the next layer, then by induction we would have to slice open the onion and understand all of physics in one fell swoop. So, although we happen to know what is inside the "hadronic layer" and the "quantum Hall layer", this is irrelevant at low energy, in both the colloquial and technical sense.

The practical value of deriving a model mathematically from a more microscopic one is that the effective coupling constants are computable in terms of the microscopic coupling constants. In a model that is constructed directly from experimental data (phenomenology), the coupling constants parametrize our residual ignorance about the microscopics. However, these are easily measured, so this does not obstruct the progress of science. In particle physics we never know anything about the next layer, but that has not stopped us from constructing the stunningly accurate Standard Model. It has more than twenty parameters that must be measured, since they cannot be calculated within this model. Could it be otherwise? The dream of deriving everything from a single mathematical structure with no free parameters remains just that, a dream, and possibly an ill-conceived one. Even if such a model does exist, it will have little or no impact on the rest of physics because of the separation of scales.

Not only all physics, but all science, is "phenomenological" or "effective" in the sense given above, and no apologies will be offered for "missing derivations". Connecting the layers directly is of course desirable, but the absence of such derivations is not a show-stopper. What is important is to have a falsifiable model (theory) that is "as simple as possible, but not simpler". There are no a priori constraints on the mathematical structures employed in the construction of a model, since it is abundantly clear that emergent phenomena may look absolutely nothing like their provenance.

As always, it is experiment that will validate a theory (or not). The stronger and more rigid the predictions following from the model, the better it is because it is easier to falsify. If experiments fail to do so (within the uncertainties of the data), this strengthens our belief in the model. And if this lack of failure continues over time, as experiments improve and error bars shrink, we are compelled to assign the model merit.

By this measure, the emergent modular symmetry identified in the quantum Hall data more than two decades ago $[6,7]$ appears to be doing very well. Not only have experiments failed to contradict the symmetry [8-10], the agreement with the coldest experiments, where the symmetry is expected to be most accurate, is now in some cases at the per mille level [11, 12].

\section{Universal data}

It is not immediately obvious that the messy diagram shown in Fig. 1(b) conceals a new type of symmetry of great power and beauty. In order to expose this symmetry we must extract those attributes of the diagram that are universal, i.e., the things that do not depend on sample geometry, immaterial material properties, etc.

We first switch to specific response functions. In two dimensions the resistances of a Hall bar of size $L_{x} \times L_{y}$, aligned with a current $I$ in the $x$-direction, are related to the resistivities $\rho^{i j}$ by $R_{* x}=$ $\left(L_{*} / L_{y}\right) \rho^{* x}$. Therefore $R_{H}=\rho^{y x}=-\rho^{x y}=\rho_{H}$, while the direct (longitudinal) resistance $R_{D}=\rho^{x x} / \square=$ $\rho_{D} / \square$ is rescaled by the aspect ratio $\square=L_{y} / L_{x}$. This transport tensor is non-trivial only because the background magnetic field breaks parity (time-reversal) invariance, whence the off-diagonal antisymmetric Hall coefficient is permitted by the generalized Onsager relation. 


\section{ICNFP 2013}

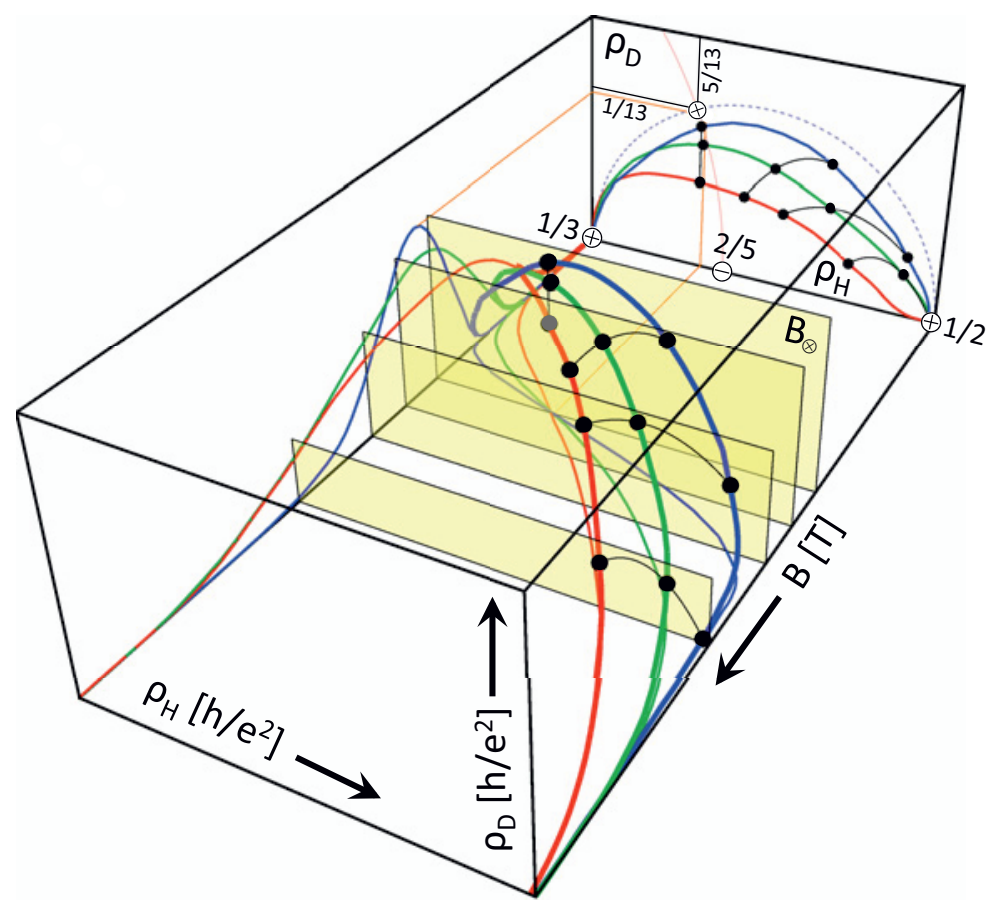

Figure 2. (Color online) Universal scaling diagrams of quantum Hall systems appear when the non-universal families of transport data are projected onto the conductivity or resistivity plane, here the transition between the second and third integer plateaux in the resistivity plane, measured at 510, 114 and $31 \mathrm{mK}$ (red is hot, blue is cold) [13]. The resulting phase and flow diagram reveals the location of fixed points, here the quantum critical point that is near the modular fixed point $\tilde{\otimes}_{3}^{\mathrm{I}}=(5+i) / 13$.

Since every term that is not forbidden by symmetry must appear in the effective theory, we expect the EFT to contain parity-violating terms. To leading order this is a topological quantity, similar to the $\theta$-term in QCD. Parametrizing the effective action by the conductivities $\sigma^{i j}=\left(\rho^{-1}\right)^{i j}$, we have schematically:

$$
\mathcal{L}_{\mathrm{eft}}=\sigma_{D} \mathcal{L}_{\mathrm{kin}}+i \sigma_{H} \mathcal{L}_{\text {top }}+\ldots
$$

This is a valuable clue for the construction of the EFT. The $O(3)$ non-linear $\sigma$-model admits such a topological term, which is important in quantum magnetism, since without it the EFT could not distinguish between ferromagnetism and antiferromagnetism. However, this model does not have the modular symmetry we find in the quantum Hall data, and the simplest viable candidate appears to be a toroidal $\sigma$-model, which admits a similar topological term.

The next step is to disentangle the two graphs $\rho_{H}(B ; T)$ and $\rho_{D}(B ; T)$, which are plotted on top of each other as functions of the magnetic field strength $B$ in Fig. 1(b), for a fixed value of the temperature $T$. We can regard these as components of a 3-dimensional parametric curve $\left(B, \rho_{H}(B), \rho_{D}(B)\right)_{T=\text { const }}$. Fig. 2 shows three such curves, plotted using experimental data for the transition between the second and third integer plateaux, obtained at $T_{1}=510 \mathrm{mK}$ (red), $T_{2}=114 \mathrm{mK}$ (green) and $T_{3}=31 \mathrm{mK}$ (blue) [13]. 


\subsection{Plateaux $(\oplus)$}

By projecting onto the "back wall" spanned by $\left(\rho_{H}, \rho_{D}\right)$ we get three curves that cut through the parameter space of the EFT, connecting the two plateaux at $\oplus=\rho_{H}=1 / 2$ and $\oplus^{\prime}=\rho_{H}^{\prime}=1 / 3$. It will be extremly convenient to label this low-energy parameter space by the complexified resistivity $\rho=$ $\rho^{x y}+i \rho^{x x}=-\rho_{H}+i \rho_{D}$, or equivalently, by the complexified conductivity $\sigma=\sigma^{x y}+i \sigma^{x x}=\sigma_{H}+i \sigma_{D}$. Except for a set of measure zero (the plateaux), these complex coordinates take values in the upper half of the complex plane ( $\mathbb{H})$, because the ordinary (direct) conductivity (resistivity) is positive. Note that the boundary of this space, which includes the plateaux, is not the real line, but only the rational numbers and "the point at infinity":

$$
\overline{\mathbb{H}}=\mathbb{H} \cup \mathbb{Q} \cup\{i \infty\}, \quad \mathbb{H}(\sigma)=\left\{\sigma \in \mathbb{C} \mid \mathfrak{J} \sigma=\sigma_{D}>0\right\} .
$$

This is the first example of a remarkable confluence of quantum Hall physics and modular mathematics, because this is precisely the space on which modular groups act. Exact Hall quantisation would therefore be an automatic consequence of a (hypothetical) modular symmetry. Our task here is to investigate this hypothesis.

\subsection{Critical points $(\otimes)$}

Notice that the three experimental curves $\rho_{H}\left(B ; T_{i}\right)(i=1,2,3)$ [13] in Fig. 2 (bottom face) all cross in a single, temperature independent critical point $\left(B_{\otimes}, \rho_{H}^{\otimes}\right)$, and that $\rho_{D}$ peaks near the same critical value $B_{\otimes}$ of the magnetic field, with the highest peak at the lowest temperature: $\max \left(\rho_{D}(B ; T)\right) \approx$ $\rho_{D}\left(B_{\otimes} ; T_{\min }\right) \stackrel{T \rightarrow 0}{\longrightarrow} \rho_{D}^{\otimes}$. We can therefore use the temperature-dependent family of quantum Hall curves $\rho(B ; T) \subset \overline{\mathbb{H}}$ to locate the quantum critical point $\tilde{\otimes}_{3}^{I}=\rho_{H}^{\otimes}+i \rho_{D}^{\otimes} \approx 0.38+0.08 i$, which controls the phase (delocalisation) transition between the phases "attached to" the integer plateaux $\oplus=1 / 2$ and $\oplus^{\prime}=1 / 3$.

Some other transitions were also investigated in [13], and Fig. 3 shows seven quantum critical points (solid red icons) obtained using data from this experiment, superimposed on the modular prediction (open blue icons) that follows from the quantum Hall group $\Gamma_{\mathrm{H}}$ identified in the next section.

\subsection{RG flow}

In fact, we can do a lot more with this family of quantum Hall curves. Since a critical point is a fixed point for scale transformations, i.e., a point that is independent of the value of the dominant scale parameter, it is clear that $T$ is this scale parameter in the data used above. If we hold all other parameters fixed, including the value of $B$, then a scale transformation will generate a temperature driven flow in parameter space, where distinct flow lines have distinct initial values given by the value of $B$ that is held constant during the flow.

A graphic visualization of this is shown in Fig. 2. A fixed $B$ plane intersects the parametric curves in three distinct points. "Connecting the dots" (quadratic interpolation was used here) and projecting the interpolating curve onto the back wall, we obtain one of the flow lines. Repeating this for a suitable set of $B$-values, we get a scaling diagram that charts part of the phase diagram. Another example, constructed using data from $[14,15]$, is shown in Fig. 4.

These phase and flow diagrams have encoded in their geometry all the universal information about the QHE, while the irrelevant, non-universal information has been projected out. In particular, the (relevant) critical correlation length exponent $v_{+}(\otimes)$ is given by the maximal (inverse) flow rate away from the critical point $\otimes$. The irrelevant exponent $v_{-}(\otimes)$, given by the maximal flow rate towards $\otimes$, 


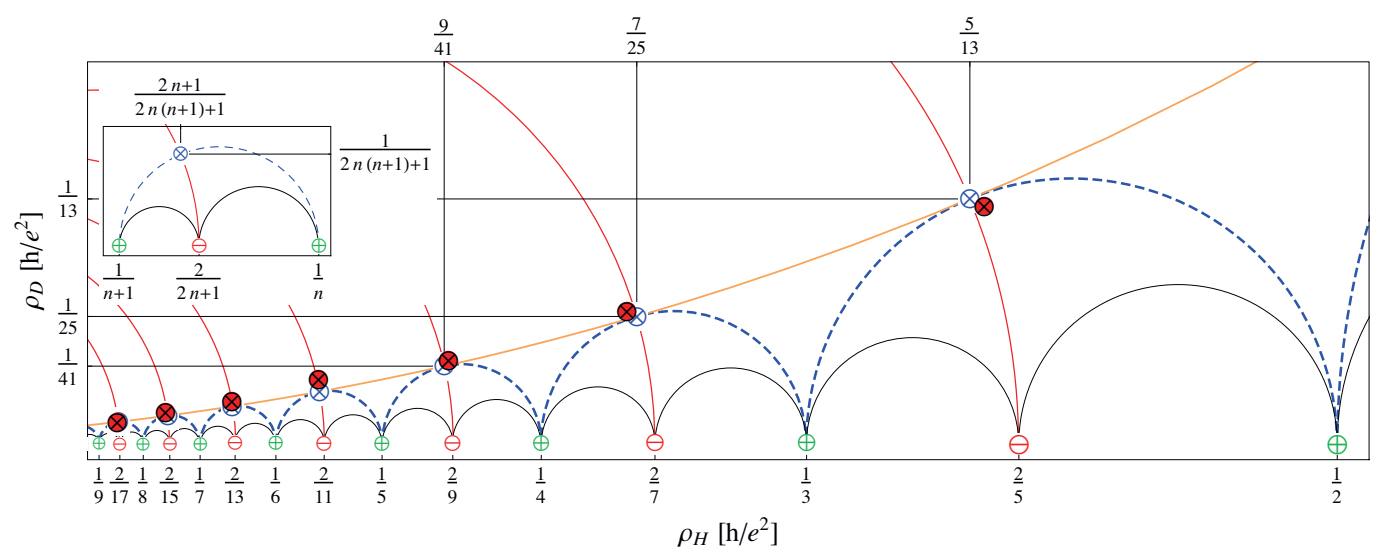

Figure 3. (Color online) Seven quantum critical points (solid red $\otimes$ ) derived from experimental data obtained in [13], which control the transitions $1 /(n+1)=\tilde{\oplus}_{n+1}^{\mathrm{I}} \longleftarrow \tilde{\otimes}_{n+1}^{\mathrm{I}} \longrightarrow \tilde{\oplus}_{n}^{\mathrm{I}}=1 / n(n=2, \ldots 8)$ discussed in Sect. 4 . Note that it is conventional to plot resistivity data in $\overline{\mathbb{H}}(-\bar{\rho})$, rather than the space $\overline{\mathbb{H}}(\rho)$ that is more natural in our theory. However, since $\rho=S(\sigma)=-\rho_{\mathrm{H}}+i \rho_{\mathrm{D}} \rightarrow-\bar{\rho}=\rho_{\mathrm{H}}+i \rho_{\mathrm{D}}$ is just a reflection in the imaginary axis, corresponding to flipping the direction of the magnetic field, this is not important here. The data are superimposed on a $\Gamma^{\mathrm{H}}$-symmetric modular phase- and flow-diagram. Inset: exact location of some theoretical critical points (open blue $\otimes$ ) following from this symmetry.

is also of interest, but much harder to measure. Distinct critical points usually have distinct critical exponents, depending on which symmetries have been broken. However, spontaneous symmetry breaking appears to have nothing to do with these phase transitions, and, lo and behold, experiments indicate that $v_{+}$takes the same value at all critical points.

Surely this means that the EFT possesses a symmetry $\Gamma_{\mathrm{H}}$ that maps the critical points into each other: $\otimes^{\prime}=\gamma(\otimes)$ for all $\gamma \in \Gamma_{\mathrm{H}}$. Together with the automatic appearance of rational plateaux $(\oplus \in \mathbb{Q})$, this leads us inexorably to consider modular transformations.

\section{Draconian symmetry}

The nested hierarchical structure that is emerging in these phase portraits is the signature of an approximate global discrete symmetry, which, given some familiarity with modular groups, is surprisingly easy to identify. No algebra beyond the manipulation of complex fractions is needed for the following discussion of fixed points ${ }^{1}$. The sources and sinks of the flow, i.e., the "trivial" ultraviolet (UV) fixed points $(\ominus)$ and infrared (IR) fixed points $(\oplus=$ plateaux), all lie on the boundary of the parameter (moduli) space. The quantum critical points $\otimes$ all lie in the interior of moduli space. This fixed point structure, which has been extracted directly from the geometry of the data, without any theoretical bias, is the DNA of the symmetry, from which all else will follow.

\subsection{The modular group $\operatorname{SL}(2, \mathbb{Z})$}

Consider first the full modular group $\operatorname{SL}(2, \mathbb{Z})=\langle T, S\rangle$, which, as an abstract group, is defined by two (and only two) algebraic constraints on the generators: $S^{2}=1=(S T)^{3}$. It can be represented

\footnotetext{
${ }^{1}$ The reader should be able to verify that if $f=(a+i b) /(c+i d) \in \mathbb{C}(a, b, c, d \in \mathbb{R})$, then $\mathfrak{R} f=(a c+b d) /\left(c^{2}+d^{2}\right)$ and $\mathfrak{I} f=(b c-a d) /\left(c^{2}+d^{2}\right)$, but no more.
} 

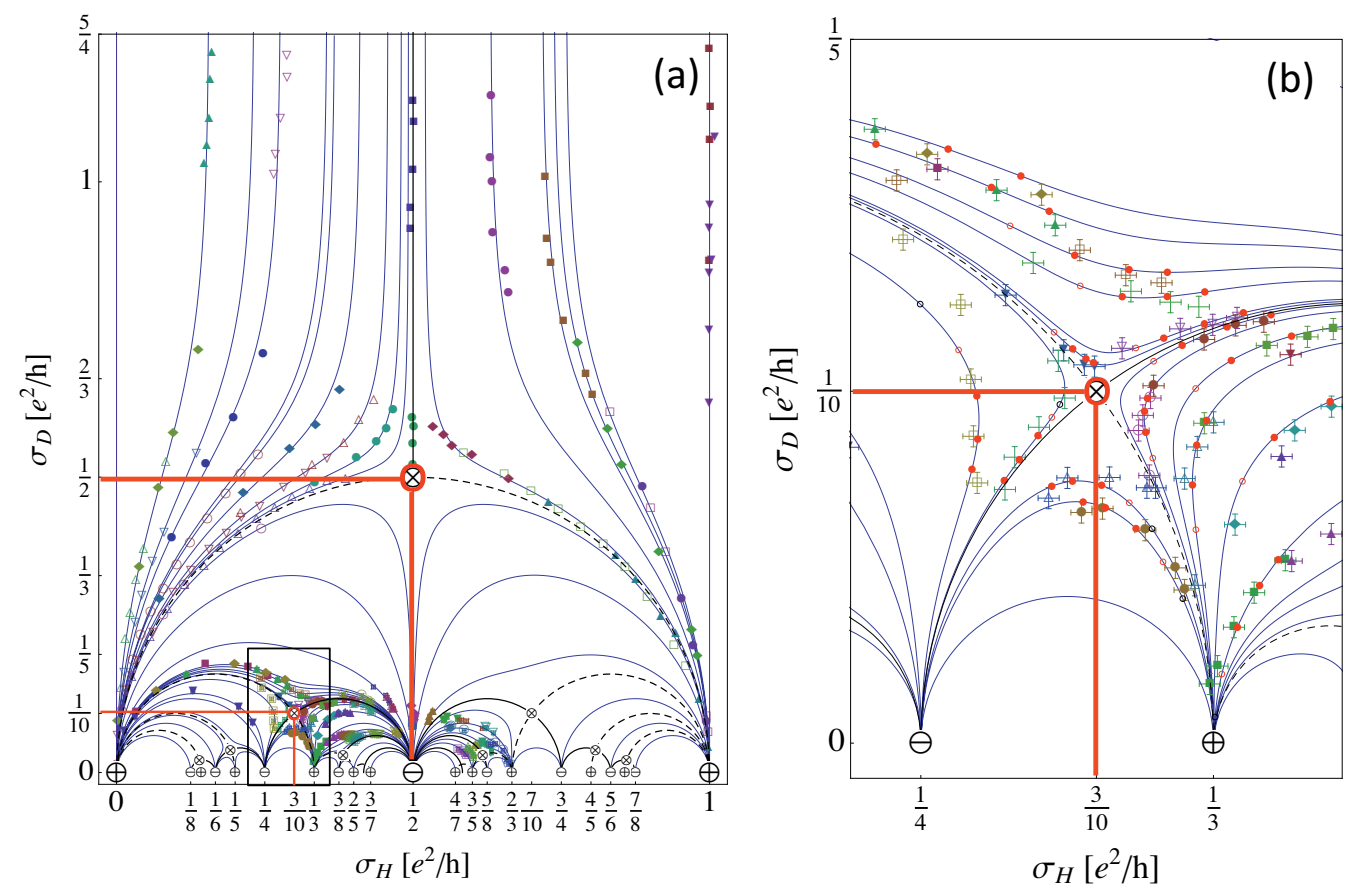

Figure 4. (Color online) A universal scaling diagram for the QHE appears when non-universal families of transport data $[14,15]$ are projected onto the conductivity plane. (a) The prototypical integer critical point $\otimes_{1}^{\mathrm{I}} \approx(0.5,0.5)$. (b) Magnification of the region enclosed by a black rectangle in (a), which contains the fractional critical point $\otimes_{3}^{\mathrm{P}} \approx(0.3,0.1)$.

by fractional linear (Möbius) transformations, generated by translations $T(z)=z+1$ and duality transformations $S(z)=-1 / z$, acting on $\overline{\mathbb{H}}(z)$.

Since $\rho=S(\sigma)$ this group acts identically on conductivity and resistivity, but this is not so for (most) subgroups, in which case we have to be clear about what $z$ is. It is the fact that $T$ and $S$ do not commute that makes this group infinite, and interesting. Any "word" in $T$ and $S$ is a fractional linear (Möbius) transformation $\gamma(z)=(a z+b) /(c z+d)$, with integer coefficients and unit determinant $(a d-b c=1)$. Because the generators satisfy two algebraic constraints there are two types of fixed points in this group: $S$ does not move the "order 2" point $i \in \mathbb{H}$, and $S T$ does not move the "order 3" point $(i \sqrt{3}-1) / 2 \in \mathbb{H}$. $\operatorname{SL}(2, \mathbb{Z})$ does not distinguish between fractions $p / q \in \mathbb{Q}$, so all rational numbers are equivalent under this symmetry. They must therefore all be of the same kind, i.e., either all repulsive UV or all attractive IR fixed points. This is in conflict with spin polarised experiments, since only odd-denominator fractions are observed, so $\operatorname{SL}(2, \mathbb{Z})$ is too strong for the QHE.

\subsection{The quantum Hall group $\Gamma_{\mathrm{H}}$}

We turn therefore to subgroups of $\operatorname{SL}(2, \mathbb{Z})$. Since the data do not exclude any integer plateaux ("fractions" that have the odd denominator 1), $T(\sigma)$ should be one of the generators of the quantum Hall group $\Gamma_{\mathrm{H}}(\sigma)$. Furthermore, if the group is too small we loose all fixed points in $\mathbb{H}(\sigma)$, which is not consistent with the existence of critical points. Surprisingly, there is only one modular group that sat- 
isfy these two, rather crude, phenomenological constraints. Acting on the conductivity it is the group $\Gamma_{\mathrm{H}}=\langle T, R\rangle$, generated by $T(\sigma)=\sigma+1$ and $R(\sigma)=S T^{-2} S(\sigma)=\sigma /(1+2 \sigma)$. This is an unnervingly effortless, robust and rigid result!

$\Gamma_{\mathrm{H}}$ partitions the parameter space into universality classes, with each phase "attached" to a unique (plateau) fixed point $\oplus$ on the real line. We have demanded that all integer plateaux exist, so some phases will converge on the integer fixed points $\oplus \in \mathbb{Z}$, but what about the rest of the odd-denominator plateaux? Does simply demanding that there exist critical points somewhere in $\mathbb{H}(\sigma)$, which together with $T$-invariance is all we used to eliminate all but one sub-modular group, force the denominators of all fractional plateaux to have the same parity? Remarkably, it does. $\Gamma_{\mathrm{H}}$ respects the parity of the denominator, because $T$ maps $p / q$ to $(p+q) / q$ and $R$ maps $p / q$ to $p /(q+2 p)$. $\Gamma_{\mathrm{H}}$ therefore partitions the rationals into two equivalence classes. Since $\Gamma_{\mathrm{H}}$ does not mix these, all even denominator rationals can be repulsive UV fixed points, while all odd denominator rationals can be attractive IR fixed points. This is exactly what we need in order to account for the spin polarised quantum Hall data (compare Fig. 4).

\subsection{Modular fixed points}

The fixed point set of $\Gamma_{\mathrm{H}}$ cannot be manipulated: all the plateaux and quantum critical points following from the group structure must be retained. Furthermore, as soon as one fixed point is pinned down, the location of all the others (of the same type) is given by the symmetry. Consider, for example, the modular transformations

$$
\alpha_{n}(z)=T^{n}(z)=z+n \quad \beta_{n}(z)=S T^{-n} S(z)=\frac{z}{1+n z} \quad(n \in \mathbb{Z}, z \in \overline{\mathbb{H}}) .
$$

\section{Fixed points in $\overline{\mathbb{H}}(\sigma)$.}

The most convenient prototype transition in $\overline{\mathbb{H}}(\sigma)$ is $\oplus_{0} \longleftarrow \otimes_{1} \longrightarrow \oplus_{1}$, where the so-called "quantum Hall insulator" $\oplus_{0}=0$ is separated from the principal plateau $\oplus_{1}=1$ by the critical point $\otimes_{1}=(1+i) / 2$ (fixed by $T R^{-1}$ ). These fixed points are observed experimentally (compare Fig. 4). Since $\alpha_{n}$ and $\beta_{2 n}$ belong to $\Gamma_{\mathrm{H}}=\left\langle\alpha_{1}, \beta_{2}\right\rangle$, for any $n \in \mathbb{Z}$, there must be plateaux and critical points in $\overline{\mathbb{H}}(\sigma)$ at:

$$
\begin{aligned}
\oplus_{n+1}^{\mathrm{I}}=\alpha_{n}\left(\oplus_{1}\right)=n+1 & \otimes_{n+1}^{\mathrm{I}}=\alpha_{n}\left(\otimes_{1}\right)=\frac{(2 n+1)+i}{2} \\
\oplus_{2 n+1}^{\mathrm{P}}=\beta_{2 n}\left(\oplus_{1}\right)=\frac{1}{2 n+1} & \otimes_{2 n+1}^{\mathrm{P}}=\beta_{2 n}\left(\otimes_{1}\right)=\frac{(2 n+1)+i}{2\left(2 n^{2}+2 n+1\right)} .
\end{aligned}
$$

The superscript $\mathrm{P}$ is used because these fractional plateaux often are referred to as the principal series. This agrees, within experimental error, with the two transitions in $\overline{\mathbb{H}}(\sigma)$ plotted in Fig. 4:

$$
\begin{aligned}
& 0=\oplus_{0} \longleftarrow \otimes_{1}^{\mathrm{I}}=(1+i) / 2 \longrightarrow \oplus_{1}^{\mathrm{I}}=1 \\
& 0=\oplus_{0} \longleftarrow \otimes_{3}^{\mathrm{P}}=(3+i) / 10 \longrightarrow \oplus_{3}^{\mathrm{P}}=1 / 3 .
\end{aligned}
$$

\section{Fixed points in $\overline{\mathbb{H}}(\rho)$.}

Since $\rho=S(\sigma)$, when the modular symmetry is acting on the resistivity it is given by the $S$-conjugate group $\Gamma^{\mathrm{H}}=\left\langle\alpha_{2}, \beta_{1}\right\rangle$. The most convenient prototype transition in $\overline{\mathbb{H}}(\rho)$ is $\tilde{\oplus}_{\infty} \longleftarrow \tilde{\otimes}_{1} \longrightarrow \tilde{\oplus}_{1}$, where the quantum Hall insulator $\tilde{\oplus}_{\infty}=i \infty$ is separated from $\tilde{\oplus}_{1}=\oplus_{1}=1$ by the critical point $\tilde{\otimes}_{1}=1+i$. Since $\alpha_{2 n}$ and $\beta_{n}$ belong to $\Gamma^{\mathrm{H}}$, for any $n \in \mathbb{Z}$, there must be plateaux and critical points in $\overline{\mathbb{H}}(\rho)$ at:

$$
\begin{array}{rlrl}
\tilde{\oplus}_{n+1}^{\mathrm{I}} & =\beta_{n}\left(\tilde{\oplus}_{1}\right)=\frac{1}{n+1} & \tilde{\otimes}_{n+1}^{\mathrm{I}} & =\beta_{n}\left(\tilde{\otimes}_{1}\right)=\frac{(2 n+1)+i}{2 n^{2}+2 n+1} \\
\tilde{\oplus}_{2 n+1}^{\mathrm{P}} & =\alpha_{2 n}\left(\tilde{\oplus}_{1}\right)=2 n+1 & \tilde{\otimes}_{2 n+1}^{\mathrm{P}}=\alpha_{2 n}\left(\tilde{\otimes}_{1}\right)=(2 n+1)+i .
\end{array}
$$


This agrees, within experimental error, with the seven transitions plotted in Fig. 3:

$$
\begin{aligned}
& 1 / 3=\tilde{\oplus}_{3}^{\mathrm{I}} \longleftarrow \tilde{\otimes}_{3}^{\mathrm{I}}=(5+i) / 13 \longrightarrow \tilde{\oplus}_{2}^{\mathrm{I}}=1 / 2 \\
& 1 / 4=\tilde{\oplus}_{4}^{\mathrm{I}} \longleftarrow \tilde{\otimes}_{4}^{\mathrm{I}}=(7+i) / 25 \quad \longrightarrow \quad \tilde{\oplus}_{3}^{\mathrm{I}}=1 / 3 \\
& 1 / 5=\tilde{\oplus}_{5}^{\mathrm{I}} \longleftarrow \tilde{\otimes}_{5}^{\mathrm{I}}=(9+i) / 41 \longrightarrow \tilde{\oplus}_{4}^{\mathrm{I}}=1 / 4 \\
& 1 / 6=\tilde{\oplus}_{6}^{\mathrm{I}} \longleftarrow \tilde{\otimes}_{6}^{\mathrm{I}}=(11+i) / 61 \longrightarrow \tilde{\oplus}_{5}^{\mathrm{I}}=1 / 5 \\
& 1 / 7=\tilde{\oplus}_{7}^{\mathrm{I}} \longleftarrow \tilde{\otimes}_{7}^{\mathrm{I}}=(13+i) / 85 \longrightarrow \tilde{\oplus}_{6}^{\mathrm{I}}=1 / 6 \\
& 1 / 8=\tilde{\oplus}_{8}^{\mathrm{I}} \longleftarrow \tilde{\otimes}_{8}^{\mathrm{I}}=(15+i) / 113 \longrightarrow \tilde{\oplus}_{7}^{\mathrm{I}}=1 / 7 \\
& 1 / 9=\tilde{\oplus}_{9}^{\mathrm{I}} \longleftarrow \tilde{\otimes}_{9}^{\mathrm{I}}=(17+i) / 145 \longrightarrow \tilde{\oplus}_{8}^{\mathrm{I}}=1 / 8 .
\end{aligned}
$$

In short, the whole fixed point structure of $\Gamma_{\mathrm{H}}$ appears to be consistent with the experimental (spin polarized) quantum Hall data.

\section{Analytical straitjacket}

How could we get so much for (apparently) so little? The answer is that we have assumed analyticity, which is a very strong constraint that ultimately must derive from the fundamental principles of quantum field theory (causality, locality, unitarity, Lorentz invariance). In our discussion above of infinite symmetries we immediately focused on holomorphic (complex analytic) Möbius functions $\gamma(z)=(a z+b) /(c z+d) \in \mathbb{C}$, both because these are intimately related to the number theory apparent in the QHE, and also because they carry the simplest representations of modular groups, which are the prototypes of these symmetries. We require complex analysis to understand just how strong this restriction is, and we need a geometric view of renormalisation to see where this complex analytic structure may come from.

\subsection{Holomorphy}

Recall first that a real analytic function $f(x \in \mathbb{R}) \in \mathbb{R}$ by definition has convergent power series expansions in open neighbourhoods of all points in its domain, and that such functions are very sparse in the set of smooth functions (functions that are infinitely differentiable. A simple example of a smooth non-analytic function is given by $f(x)=\{0, x \leq 0$; $\exp (-1 / x), 0<x\}$. While $f$ is smooth everywhere, it has no power series expansion near $x=0$. So real analyticity is already a powerful constraint, weeding most smooth functions, but by restricting attention to functions of a complex variable $z=x+i y$ we have actually assumed much more.

Möbius transformations are holomorphic, which means that they are complex differentiable. This is a severe restriction, since the derivative $f^{\prime}=\lim _{z \rightarrow z_{0}} \delta f / \delta z$ at $z_{0}$ must be well defined (existence and uniqueness) for all possible limiting procedures that take $z$ to $z_{0}$ (both in $\mathbb{C}$ ). By the fundamental theorem of complex analysis a function is holomorphic iff it is complex analytic, so these functions automatically have convergent complex power series expansions, and are therefore smooth. A holomorphic function depends only on $z=x+i y$. It is therefore a constant function of $\bar{z}=x-i y$, so $\bar{\partial} f=0$, where $\partial=\partial / \partial z=\left(\partial_{x}-i \partial_{y}\right) / 2$ and $\bar{\partial}=\partial / \partial \bar{z}=\left(\partial_{x}+i \partial_{y}\right) / 2$. Since the real and imaginary parts of this equation must both vanish, $\bar{z}$-independence is equivalent to the more familiar CauchyRiemann equations for the real functions $u(x, y)=\mathfrak{R} f(x, y)$ and $v(x, y)=\mathfrak{J} f(x, y): \partial_{x} u-\partial_{y} v=0$ and $\partial_{x} v+\partial_{y} u=0$.

In other words, we have only considered holomorphic functions, and this is so restrictive that a modular symmetry barely manages to satisfy the phenomenology. The fact that this is all possible, and in detailed agreement with experiments, is ample motivation to search for a physical principle behind the complex analytical structure. 
ICNFP 2013

\subsection{RG geometry}

Renormalization is a profoundly geometric procedure if we assume, as we always do in quantum field theory, that the moduli space $\mathcal{M}$ of the EFT is a Riemannian manifold, i.e., a smooth space equipped with a connection and metric. For each starting point in $\mathcal{M}$ the renormalisation group (RG) generates a continuous, smooth path through the space of renormalised coupling constants, i.e., a streamline. The collection of all these curves is a geometric flow. We argue that the RG flow on the moduli space of the QHE is Laplacian, i.e., both incompressible (divergence free) and irrotational (curl free). A Laplacian flow in two dimensions is automatically holomorphic.

At each point in $\mathcal{M}$ where the $\mathrm{RG}$ flow is finite, the flow rates are components of a vector tangent to the flow. This gives a vector field $\beta$ on $\mathcal{M}$ that contains the universal data. The global (topological) structure of this flow contains information about the non-perturbative structure of the EFT, and is therefore not accessible by perturbation theory. However, the phenomenology does of course contain this information, and we have seen that the quantum Hall data suggests that the RG flow is nonsingular on $\mathcal{M}$, except at fixed points $\otimes$ of $\Gamma_{\mathrm{H}}$ where the flow stops, i.e., at critical points of the RG flow where $\beta(\otimes)=0$. This means that there are no sources or sinks on $\mathcal{M}$, so the flow is conserved. The vector field $\beta=(u, v)$ is therefore divergence free, with continuity equation $\partial_{x} u+\partial_{y} v=0$.

The physical interpretation of an RG flow is that the number of effectively massless degrees of freedom decreases monotonically as the energy decreases, so the flow is irreversible. The flow should be irrotational so that flow lines can not close, since limit cycles would render this interpretation meaningless. Consequently, we expect an RG flow to be conservative, i.e., a gradient flow $\beta=\operatorname{grad} \varphi$ generated by an RG potential $\varphi$, in which case the RG flow is curl free, $\partial_{x} v-\partial_{y} u=0$. The RG potential is often called a $C$-function, following Zamolodchikov [17] who proved that it exists in two dimensional theories. Friedan's proof of the $C$-theorem [18] emphasizes unitarity, and our argument inherits this property. Unitarity is therefore one of the roots of holomorphy in the QHE.

Changing coordinates to $(x, \bar{y}=-y)$ we recover the Cauchy-Riemann equations, and by the fundamental theorem of complex analysis the vector field is therefore holomorphic in $x+i \bar{y}=x-i y=\bar{z}$, i.e., anti-holomorphic in $z$. In other words, if the phenomenological observation (incompressible flow) and conventional physical intuition (irrotational flow) are correct, then the flow is forced into the analytical straitjacket implicitly used in our holomorphic discussion of fixed points in the previous section. Holomorphic modular forms can (and do) have singularities only on the boundary of the compactified moduli space $\overline{\mathcal{M}}$.

\subsection{Automorphy}

When $\overline{\mathcal{M}}$ has a symmetry group $\Gamma$ that commutes with the RG, this forces the flow rate at $z \in \mathcal{M}$ and at any image point $z^{\prime}=\gamma(z)=(a z+b) /(c z+d) \in \mathcal{M}(\gamma \in \Gamma)$ to be equal. This is a severe constraint on the tangent vector field, which if it is contravariant transforms as $\beta\left(z^{\prime}\right)=\left(\partial z^{\prime} / \partial z\right) \beta(z)=(c z+d)^{-2} \beta(z)$, and if it is covariant transforms as $\beta\left(z^{\prime}\right)=\left(\partial z / \partial z^{\prime}\right) \beta(z)=(c z+d)^{2} \beta(z)$. In group theory a holomorphic function that transforms like this is called a modular (automorphic) form of weight $w= \pm 2$. The fundamental theorem of modular mathematics is that there are no modular forms of negative weight, so it is the covariant $\beta$-function that can potentially be a modular form. In fact, there are no forms of weight $w=2$ on the full modular group $\operatorname{SL}(2, \mathbb{Z})$. Again we are forced to consider subgroups if modular symmetry constrains the phase and RG flow diagram.

In another example of the impressive convergence of quantum Hall physics and modular mathematics, there is a unique modular form (up to normalization) of weight $w=2$ on $\Gamma_{\mathrm{H}}$, which therefore 


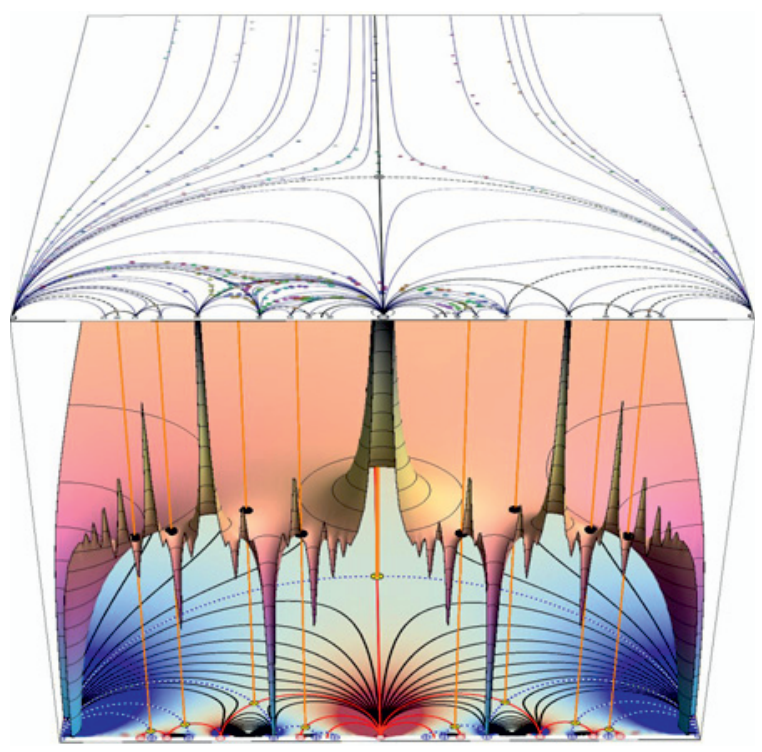

Figure 5. (Color online) The $\Gamma_{\mathrm{H}}$-symmetric RG potential $|\varphi|$, with some gradient flow lines (top and bottom face of cube), and the same experimental data shown in Fig. 4 (top face).

is our candidate $\beta$-function for the (spin polarized) QHE:

$$
\beta(\sigma) \propto \partial \varphi(\sigma), \quad \varphi(\sigma)=\ln \eta(2 \sigma)-\ln \eta(\sigma), \quad \eta(\sigma)=q^{1 / 24} \prod_{n=1}^{\infty}(1-q)^{n} \quad\left(q=e^{2 \pi i \sigma}\right) .
$$

The Dedekind $\eta$-function $\eta(\sigma)$ is subtle but extremely well studied, since it figures prominently in many parts of mathematics. Notice that this is indeed a gradient flow. It is the flow lines of this potential that have been plotted in our diagrams, compare Fig. 5. There is a remarkable agreement between the shapes of the flow lines derived from $\varphi$ and experimental temperature driven flow data, see Fig. 4.

\subsection{Critical exponents}

The final pièce de résistance in the QHE is to compute the critical exponents. Our first result is that the modular symmetry and complex structure uncovered in the data means that our theory only has one critical (correlation length) exponent: $v_{+}(\otimes)=-v_{-}(\otimes)$, for any critical point $\otimes=\gamma\left(\otimes_{1}\right)\left(\gamma \in \Gamma_{\mathrm{H}}\right)$. This super-universality of $v_{+}$has been found experimentally [16]. The "anti-holomorphic" scaling giving $v_{+}=-v_{-}$is not inconsistent with numerical or real experiments, but since the irrelevant exponent is notoriously hard to determine this is not a severe test.

The value of the exponent is not given by symmetry alone. Just as in the analogous case of orderdisorder-duality (a $\mathbb{Z}_{2}$ symmetry) in the Ising model, which allowed Kramers and Wannier to find the exact location of the critical point, it was only by solving the dynamical problem that Onsager could determine the exact value of the exponent. 
However, the modular symmetry does narrow the search for an EFT enormously. The simplest scenario is arguably a toroidal $\sigma$-model with $m$ fermions $[6,12]$. With a specific spin structure on the toroidal target space the partition function for this family of models has the required modular symmetry $\Gamma_{\mathrm{H}}$. We find critical exponents $v_{m}=(n / m) v_{\text {tor }}$, where $n$ determines the normalisation of the hyperbolic (Zamolodchikov) metric on moduli space. The value of $v_{\text {tor }}=18 \ln 2 / \pi^{2} G^{4} \approx 2.6051 \cdots \approx$ $21 / 8$ ( $G$ is Gauss' constant) is in remarkable agreement with the best available value from numerical simulations of the quantum Hall transition, $v_{\text {num }}=2.607 \pm 0.004$ [19].

\section{Summary}

We have seen how to project out the universal data contained in experimental quantum Hall response curves, and used the geometry of the resulting RG flow diagram to find some quantum critical points in the conductivity (or resistivity) plane. This is sufficient to identify a unique modular symmetry $\Gamma_{\mathrm{H}}$ hiding in the data. The flow is incompressible, because the phenomenology expels all sources and sinks to the boundary of moduli space. General principles of quantum field theory require the RG flow to also be irrotational. The flow is therefore a holomorphic, automorphic (modular), gradient flow. These constraints are so strong that they eliminate all but one $\beta$-function, whose zeros are pinned to a rigid fractal hierarchy of quantum critical points. The location of these fixed points are in close agreement with available scaling data. The simplest family of $\Gamma_{\mathrm{H}}$-invariant models (toroidal $\sigma$-models) contains a critical exponent that agrees with the best available numerical value at the per mille level. The emergent global symmetry $\Gamma_{\mathrm{H}}$ automatically gives Hall quantization, which if $\Gamma_{\mathrm{H}}$ were exact $(T \rightarrow 0)$ would be rational.

\section{References}

[1] E.H. Hall, Am. J. Math. 2, 287 (1879)

[2] K.v. Klitzing, G. Dorda, M. Pepper, Phys. Rev. Lett. 45, 494 (1980)

[3] D.C. Tsui, H.L. Störmer, A.C. Gossard, Phys. Rev. Lett. 48, 1559 (1982)

[4] H.L. Störmer, Rev. Mod. Phys. 71, 875 (1999)

[5] S. Weinberg, Physica 96A, 327 (1979)

[6] C.A. Lütken, G.G. Ross, Phys. Rev. B 45, 11837 (1992); Phys. Rev. B 48, 2500 (1993)

[7] C.A. Lütken, Nucl. Phys. B 396, 670 (1993); J. Phys. A 26, L811 (1993)

[8] C. P. Burgess, C.A. Lütken, Nucl. Phys. B 500, 367 (1997); Phys. Lett. B 451, 365 (1999)

[9] C.A. Lütken, Nucl. Phys. B 759, 343 (2006)

[10] C.A. Lütken, G.G. Ross, Phys. Lett. A 356, 382 (2006); Phys. Lett. A 374, 4700 (2010)

[11] C.A. Lütken, G.G. Ross, Nucl. Phys. B 850, 321 (2011)

[12] C.A. Lütken, G.G. Ross, Phys. Lett. B 653, 363 (2007); Phys. Lett. A 378, 262 (2014)

[13] W. Li, G.A. Csathy, D.C. Tsui, L.N. Pfeiffer, K.W. West, Phys. Rev. Lett. 94, 206807 (2005)

[14] S.S. Murzin, M. Weiss, A.G.M. Jansen, K. Eberl, Phys. Rev. B 66, 233314 (2002)

[15] S.S. Murzin, S.I. Dorozhkin, D.K. Maude, A.G.M. Jansen, Phys. Rev. B 72, 195317 (2005)

[16] S. Koch, R.J. Haug, K.v. Klitzing, K. Ploog, Phys. Rev. Lett. 67, 883 (1991); Phys. Rev. B 46, 1596 (1992)

[17] A.B. Zamolodchikov, JETP Lett. 43, 730 (1986)

[18] A. Cappelli, D. Friedan, J.I. Latorre, Nucl. Phys. B 352, 616 (1991)

[19] K. Slevin,T. Ohtsuki, Int. J. Mod. Phys. Conf. Ser. 11, 60 (2012) 